\title{
Species Transferability of Klebsiella pneumoniae Carbapenemase-2 Isolated from a High-Risk Clone of Escherichia coli ST410
}

\author{
Miyoung Lee ${ }^{1,2}$ and Tae-Jin Choi ${ }^{1 *}$ \\ ${ }^{1}$ Department of Microbiology Pukyoung National University Busan 48513, Republic of Korea \\ ${ }^{2}$ Department of Laboratory Medicine, BHS Hanseo Hospital Busan 48253, Republic of Korea
}

\begin{abstract}
Sequence type 410 (ST410) of Escherichia coli is an extraintestinal pathogen associated with multi drug resistance. In this study, we aimed to investigate the horizontal propagation pathway of a highrisk clone of E. coli ST410 that produces Klebsiella pneumoniae carbapenemase (KPC). blaKPCencoding $E$. coli and $K$. pneumoniae isolates were evaluated, and complete sequencing and comparative analysis of blaKPC-encoding plasmids from $E$. coli and $K$. pneumoniae, antimicrobial susceptibility tests, polymerase chain reaction, multilocus sequence typing, and conjugal transfer of plasmids were performed. Whole-genome sequencing was performed for plasmids mediating KPC-2 production in E. coli and K. pneumoniae clinical isolates. Strains E. coli CPEc171209 and K. pneumoniae CPKp171210 were identified as ST410 and ST307, respectively. CPEc171209 harbored five plasmids belonging to serotype $08: \mathrm{H} 21$, which is in the antimicrobial-resistant clade C4/H24. The CPKp171210 isolate harbored three plasmids. Both strains harbored various additional antimicrobial resistance genes. The IncX3 plasmid pECBHS 9 5 harbored blaKPC-2 within a truncated Tn4401a transposon, which also contains blaSHV-182 with duplicated conjugative elements. This plasmid displayed $100 \%$ identity with the IncX3 plasmid pKPBHS_10_3 from the $K$. pneumoniae CPKp171210 ST307 strain. The genes responsible for the conjugal transfer of the IncX3 plasmid included tra/trb clusters and pil genes coding the type IV pilus. ST410 can be transmitted between patients, posing an elevated risk in clinical settings. The emergence of a KPC-producing $E$. coli strain (ST410) is concerning because the blaKPC-2-bearing plasmids may carry treatment resistance across species barriers. Transgenic translocation occurs among carbapenem-resistant bacteria, which may spread rapidly via horizontal migration.
\end{abstract}

Keywords: Escherichia coli ST410, Klebsiella pneumoniae ST307, extraintestinal pathogenic E. coli, blaKPC-2, Tn4401a

Received:December 29, 2019 Accepted: May 19, 2020

First published online: May 21, 2020

* Corresponding author Phone: +82-51-629-5617 Fax: +82-51-629-5619 E-mail: choitj@pknu.ac.kr

pISSN 1017-7825 elSSN $1738-8872$

Copyright(C) 2020 by The Korean Society for Microbiology and Biotechnology

\section{Introduction}

The gram-negative bacteria family Enterobacteriaceae includes pathogens that are responsible for a large number of infections and deaths worldwide each year. Furthermore, the continuously increase in the prevalence of antibiotic resistance in this bacteria family poses a serious problem [1]. Carbapenemases that endow bacteria with carbapenem resistance often jeopardize allopathic treatment for infectious diseases caused by common nosocomial pathogens. Currently, Klebsiella pneumoniae carbapenemases (KPCs), belonging to the Ambler class A carbapenemases, are the most clinically concerning enzymes because of their global presence and capability of using a broad spectrum of substrates, including most $\beta$-lactam drugs, except cephamycins $[2,3]$. Molecular epidemiological studies on multilocus sequence typing (MLST) for KPC-producing Escherichia coli have revealed 131 more widespread strains than the ST410, ST69, ST93, ST167, ST354, and ST3948 strains based on their sequence type (ST) [4-7]. E. coliST 410 has been reported worldwide as an extraintestinal pathogen and associated with resistance to fluoroquinolones, third-generation cephalosporins, and carbapenems. Recent studies on ten extended-spectrum $\beta$-lactamase (ESBL)-producing E. coli ST410 isolates from Germany indicated that this lineage includes a new successful clone with cross-sectorial transmission among wildlife, humans, pets, and animals used for commercial purposes through various environmental modes [8,9]. The accumulation of multidrug resistance in E. coli ST410 over the past two decades, along with its potential for transmission between patients, indicates its high risk in clinical settings [10]. Previous studies on carbapenemase-producing Enterobacteriaceae (CPE) among inpatients demonstrated the clonal spreading of CPE between patients [11, 12]. 
The transposon Tn4401, which is based on highly mobile Tn3, facilitates the dissemination of blaKPC [13], potentially resulting in horizontal gene transfer to other bacterial species. As the mortality rate associated with invasive infections caused by CPE is high [14], the increasing incidence of carbapenem-resistant bacteria, which are resistant to nearly all antibiotics, is of great concern [15]. This study was performed to investigate the horizontal propagation pathway of the KPC-producing high-risk clone of E. coli ST410 in one patient. The mechanism and KPC delivery pathways based on the acquisition of multidrug resistance, including resistance to carbapenem, were investigated using whole-genome sequencing. This study elucidated the risk of this $E$. coli clone to spread genes involved in antibiotic resistance across species, providing clues that can be integrated in the management of high-risk clones in South Korea.

\section{Materials and Methods \\ Patient description}

A 79-year-old man with chronic kidney disease was admitted to a general hospital (Busan, Korea) on December 12, 2017, owing to kidney disease. Upon admission, a KPC-2 E. coli isolate (CPEc171209) was detected in rectal swabs, and on December 21, 2017, the 9th day of hospitalization, KPC-2 K. pneumoniae (CPKp171210) was isolated. This prospective study was conducted under the approval of the Institutional Review Board of the BHS Hanseo Hospital, Busan, Korea (approval number CTS-19-002).

\section{Bacterial Isolates and Antimicrobial Susceptibility Testing}

We evaluated the clinical strains of E. coli (CPEc171209) and K. pneumoniae (CPKp171210) isolated from the rectal swabs harvested from the patient. Bacteria were identified through standard microbiological procedures and VITEK-2 (bioMérieux, Marcy-l'Etoile, France). The identification of all isolates was confirmed through 16S rDNA sequencing [16]. VITEK 2 AST N224 cards (bioMérieux) and disk diffusion tests on Mueller-Hinton agar (BD Biosciences, Franklin Lakes, NJ, USA) were used to determine antimicrobial susceptibilities in accordance with the Clinical and Laboratory Standards Institute (CLSI) guidelines [17]. The susceptibility test was conducted with ampicillin, aztreonam, cefotaxime, ceftazidime, cefoxitin, ciprofloxacin, amikacin, gentamicin, imipenem, meropenem, ertapenem, and colistin. The minimum inhibitory concentrations (MIC) for colistin were determined using the broth microdilution method with Mueller-Hinton broth (BD Biosciences) in accordance with the European Committee on Antimicrobial Susceptibility Testing guidelines [18]. The susceptibility results for tigecycline were confirmed through the E-test (bioMérieux).

For the modified carbapenem inactivation method (mCIM) test, organisms were incubated with a meropenem disk in tryptic soy broth (Bacto Laboratories, Mount Pritchard, Australia). For the ethylene diamine tetra acetic acid (EDTA)-mCIM (eCIM) test, EDTA was added to the broth to chelate metal ions required for metallo- $\beta$ lactamase function. After incubation, the disks were removed and placed on a lawn of susceptible E. coli (ATCC 25922) to determine whether the test organisms degrade meropenem. Clearance zones were measured and interpreted according to the CLSI guidelines [17].

\section{Genotyping of $\beta$-Lactamases and Outer Membrane Proteins}

The $\beta$-lactamase-encoding gene was selected through polymerase chain reaction (PCR). Genes encoding carbapenemases (IMP-1-type, VIM-2-type, NDM, KPC, KPC-2, GES, and OXA-48-like) and extended-spectrum $\beta$-lactamases (CTX-M-1-, CTX-M-9-, TEM-, and SHV-type), were assessed by PCR as previously described [19]. $\mathrm{CPE}$ isolates were examined for the presence of $16 \mathrm{~S}$ ribosomal methyltransferases ( $\mathrm{armA}, \mathrm{rmtA}, \mathrm{rmtB}$, and $\mathrm{rmtD}$ ) $[20,21]$, and quinolones (qepA, qnrA, qnrB, and qnrS), as previously described [22], and of genes encoding outer membrane proteins (ompK35 and ompK36) [23].

\section{MLST}

PCR and sequencing of the amplified DNA fragments were performed for seven housekeeping genes: $a d k$, fum $C, g y r B$, icd, $m d h$, purA, and recA of E. coli [24] and gapA, infB, $m d h, p g i$, phoE, rpoB, and tonB of $K$. pneumoniae [25]. Nucleotide sequences were compared to those available in the MLST database (http:// mlst.warwick.ac.uk/mlst/dbs/Ecoli) to identify allelic numbers and STs.

\section{Bacterial Conjugation}

Bacterial conjugation was conducted using the E. coli strain CPEc171209 and Klebsiella pneumoniae CPKp171210 strains as donors and sodium azide-resistant $E$. coli 553 strain as the recipient, following a standard agar mating method [26]. After overnight incubation at $37^{\circ} \mathrm{C}$ on brain-heart infusion agar (MB Cell, USA), transconjugants were selected on brain-heart infusion agar supplemented with $100 \mu \mathrm{g} / \mathrm{ml}$ sodium azide and $0.5 \mu \mathrm{g} / \mathrm{ml}$ imipenem.

\section{Whole-Genome Sequencing}

Whole-genome sequencing of CPEc171209 and CPKp171210 was performed. Single-molecule real-time sequencing was performed using a PacBio RSII instrument (Pacific Biosciences, USA). Schematic diagrams of the multiple alignments of plasmids were generated manually by realigning the linear plasmid maps generated using the SnapGene Viewer software (http://www.snapgene.com/products/snapgene viewer/). Prokka 1.11 (http:// www.vicbioinformatics.com/software.prokka.shtml) was used for sequence annotation. ResFinder (https:// cge.cbs.dtu.dk/services/ResFinder/), IS-Finder (https://isfinder.biotoul.fr/), Plasmid Finder (https://cge.cbs.dtu.dk/ services/PlasmidFinder/), Virulence Factor Database (http://www.mgc.ac.cn/VFs/), Restriction-Modification 
Finder (https://cge.cbs.dtu.dk/services/Restriction-ModificationFinder/), Serotype Finder (https://cge.cbs.dtu.dk/ services/SerotypeFinder/), Fim Typer (https://cge.cbs.dtu.dk/services/FimTyper/), CH Typer (https://cge.cbs.dtu.dk/ services/CHTyper/), and TA Finder 1.0 (http://202.120.12.133/TAfinder/index.php) were used to identify resistance genes, insertion elements, replication origins, virulent elements, and toxin and antitoxin systems, respectively.

\section{Data Availability}

GenBank accession numbers for the two sequenced genomes are WMHS01000001-WMHR01000006 (CPEc171209) and WMHR01000001-WMHR01000004 (CPKp171210).

\section{Results}

\section{Antimicrobial Susceptibilities and Molecular Typing}

The antimicrobial susceptibility profiles of the E. coli and K. pneumoniae strains are presented in Table 1. According to the antibiotic susceptibility profiles, both isolates were resistant to ampicillin, aztreonam, cefotaxime, ceftazidime, cefoxitin, ciprofloxacin, gentamicin, imipenem, meropenem, and ertapenem but susceptible to amikacin, tigecycline, and colistin. Carbapenemase susceptibility tests revealed that both isolates were resistant (Table 2). Furthermore, transconjugant strains displayed multidrug resistance (MDR) patterns during drug susceptibility assays, albeit with reduced cephalosporin and aminoglycoside resistance (Table 3). Differentiation tests for Phenotypic carbapenemase were positive for KPC production in both isolates. PCR of $\beta$ lactamase genes and sequence analysis of the resulting products supported the presence of blaKPC-2 and blaSHVlike in both isolates. Also, ompK36 was obliterated from CPKp171210.

\section{Sequencing and Annotation of CPEc171209}

The E. coli strain CPEc171209 genome comprised 5,293,485-bp with a 4,787,633-bp chromosome and five plasmids. They harbored the virulence factors gad and lpfA as well as S83L and D87N substitutions in gyrA, an S80I substitution in parC, an S458A substitution in parE, and unknown mutations in parC, pmrA, 23S, 16S_rsC, pmrB, 16S_rsH, and $16 S \_r s B$. The type II restriction enzyme $M . E c o J A 03 P D c m$, and M.EcoGVI genes were

Table 1. Antimicrobial susceptibilities and epidemiological properties of KPC-producing Enterobacteriaceae isolates ${ }^{\mathrm{a}}$.

\begin{tabular}{|c|c|c|c|c|c|c|c|c|c|c|c|c|c|}
\hline \multirow{3}{*}{ Isolate ID } & \multirow{3}{*}{ Specimen } & \multirow{3}{*}{ Date } & \multirow{3}{*}{$\begin{array}{c}\text { MLST } \\
\text { ST }\end{array}$} & \multicolumn{2}{|c|}{ blaKPC } & \multicolumn{6}{|c|}{ Antibiotic susceptibility ${ }^{\mathrm{b}}$} & $\begin{array}{c}\text { Carbapenemase } \\
\text { differentiation } \\
\text { test }^{c}\end{array}$ & \multirow{3}{*}{$\begin{array}{l}\text { Porin } \\
\text { loss }\end{array}$} \\
\hline & & & & Subtype & $\begin{array}{l}\text { Bracketed } \\
\text { by }\end{array}$ & \multicolumn{4}{|c|}{ MIC (mg/L) } & \multicolumn{2}{|c|}{$\begin{array}{l}\text { Zone } \\
\text { diameter } \\
(\mathrm{mm})\end{array}$} & mCIM eCIM & \\
\hline & & & & & & $\mathrm{AK}$ & GN & CST & TIG & $\mathrm{AK}$ & CST & & \\
\hline СРEc171209 & Rectal & $\begin{array}{c}\text { Dec 12, } \\
2017\end{array}$ & 410 & blaKPC-2 & $\Delta \operatorname{Tn} 4401 a$ & 4 & - & 0.25 & 0.075 & 20 & 12 & + & - \\
\hline СРКр171210 & Rectal & $\begin{array}{c}\text { Dec 21, } \\
2017\end{array}$ & 307 & blaKPC-2 & $\Delta \operatorname{Tn} 4401 a$ & $\leq 2$ & $\leq 1$ & 0.25 & 0.25 & 20 & 14 & + & ompK36 \\
\hline
\end{tabular}

${ }^{a}$ The breakpoints were applied according to the Clinical and Laboratory Standards Institute (CLSI) guidelines. Tigecycline susceptibility was confirmed by E-test (bioMérieux), and colistin susceptibility was confirmed by broth microdilution.

${ }^{b}$ Disk diffusion test results were interpreted according to CLSI guidelines. The results for colistin are not shown because of the lack of suggested breakpoints.

${ }^{c}$ The eCIM is only interpreted when the mCIM result is positive. In contrast to MCIM, when the eCIM result is interpreted, pinpoint colonies within the zone of growth inhibition around the meropenem disk incubated in the presence of EDTA should be ignored. An indeterminate mCIM result occurs when the zone size is $16-18 \mathrm{~mm}$, when the zone size is $\geq 19 \mathrm{~mm}$ with pinpoint colonies in the zone of growth inhibition, or when carbapenemase production cannot be confirmed. An eCIM zone size of $16-$ $18 \mathrm{~mm}$ with pinpoint colonies in the zone of growth inhibition is also considered a positive mCIM result.

Abbreviations: AK, amikacin; GN, gentamicin; CST, colistin; TIG, tigecycline; mCIM, modified carbapenem inactivation method; eCIM, EDTA-modified carbapenem inactivation method.

Table 2. Carbepenem susceptibility profiles of KPC-producing Enterobacteriaceae isolates ${ }^{\mathrm{a}}$.

\begin{tabular}{cccccccccc}
\hline & \multicolumn{4}{c}{ MIC $(\mathrm{mg} / \mathrm{L})$} \\
\cline { 2 - 8 } Antibiotics & CPEc & CPKp & TCPEc & TCPKp & CPEc & CPKp & TCPEc & TCPKp & Interpretation \\
& 171209 & 171210 & 171209 & 171210 & 171209 & 171210 & 171209 & 171210 & \\
\hline IMP & $\geq 16$ & $\geq 16$ & $\geq 16$ & $\geq 16$ & 0 & 0 & 0 & 0 & $\mathrm{R}$ \\
MEM & - & - & - & - & 0 & 0 & 0 & 0 & $\mathrm{R}$ \\
ETP & $\geq 8$ & $\geq 8$ & $\geq 8$ & 4 & 0 & 0 & 0 & 0 & $\mathrm{R}$ \\
\hline
\end{tabular}

${ }^{\mathrm{a}} \mathrm{CPEc} 171209$ and CPKp171210 are pre-conjugation strains, and TCPEc171209 and TCPKp171210 are the conjugated strains. Abbreviations: IMP, imipenem; MEM, meropenem; ETP, ertapenem. 
Table 3. Antibiotic susceptibility profiles of KPC-producing Enterobacteriaceae isolates ${ }^{\text {. }}$

\begin{tabular}{lcccccccccccccccccc}
\hline \multirow{2}{*}{ Isolate ID $^{\mathrm{b}}$} & \multicolumn{10}{c}{ MIC $(\mathrm{mg} / \mathrm{L})$} \\
\cline { 2 - 7 } & AMP & AMOX-CLA & TZP & CFZ & FOX & CTX & CAZ & FEP & ATM & ETP & IMP & AK & GN & CIP & SXT & TIG & CST \\
\hline CPEc171209 & $\geq 32$ & $\geq 32$ & $\geq 128$ & $\geq 64$ & $\geq 64$ & $\geq 64$ & $\geq 64$ & $\geq 64$ & $\geq 64$ & $\geq 8$ & $\geq 16$ & 4 & $\geq 16$ & $\geq 4$ & $\leq 0.5$ & 0.075 & 0.25 \\
CPKp171210 & $\geq 32$ & $\geq 32$ & $\geq 128$ & $\geq 64$ & 16 & 8 & 16 & 2 & $\geq 64$ & $\geq 8$ & $\geq 16$ & $\leq 2$ & $\leq 1$ & $\geq 4$ & 2 & 0.25 & 0.25 \\
\hline TCPEc171209 & $\geq 32$ & $\geq 32$ & $\geq 128$ & $\geq 64$ & $\geq 64$ & $\geq 64$ & $\geq 64$ & $\geq 64$ & $\geq 64$ & $\geq 8$ & 8 & 4 & $\geq 16$ & $\geq 4$ & $\leq 0.5$ & 0.075 & 0.25 \\
TCPKp171210 & $\geq 32$ & $\geq 32$ & $\geq 128$ & $\geq 64$ & 32 & 8 & 16 & 2 & $\geq 64$ & 4 & $\geq 16$ & $\leq 2$ & $\leq 1$ & $\geq 4$ & $\leq 0.5$ & 0.25 & 0.25 \\
\hline
\end{tabular}

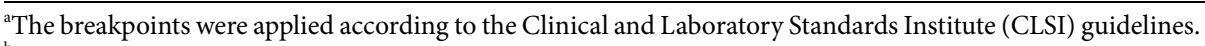

${ }^{\mathrm{b}} \mathrm{CPEc} 171209$ and CPKp171210 are pre-conjugation strains, and TCPEc171209 and TCPKp171210 are the conjugated strains.

Abbreviations: AMP, ampicillin; AMOX-CLA, amoxicillin/clavulanic acid; TZP, piperacillin/tazobactam; CFZ, cefazolin; FOX, cefoxitin; CTX, cefotaxime; CAZ, ceftazidime; FEP, cefepime; ATM, aztreonam; ETP, ertapenem; IPM, imipenem; AK, amikacin; GN, gentamicin; CIP, ciprofloxacin; SXT, trimethoprim/sulfamethoxazole; TIG, tigecycline; CST, colistin. MIC; minimum inhibitory concentration.

detected. CPEc171209 belonged to ST410, the serotype belonged to STO8, and H21 and its subtype belonged to fum $\mathrm{C} 4$ and fim $\mathrm{H} 24$, respectively. Furthermore, outer membrane proteins $\mathrm{F}$ and $\mathrm{C}$ were detected. Chromosomes included $m p h$ (A) for macrolide resistance. The 90,979-bp plasmid (pECBHS_9_3) and 85,870-bp plasmid (pECBHS_9_4) did not contain any acquired antimicrobial resistance factors.

pECBHS_9_1 plasmid composition. The pECBHS_9_1 plasmid consisted of a 188,153-bp circular DNA molecule with an average $\mathrm{G}+\mathrm{C}$ content of $51.1 \%$ and 126 annotated open reading frames (ORFs).

The IncA/C2 plasmid (pECBHS_9_1) carried blaCTX-M-14 and blaTEM-1C for $\beta$-lactam resistance, aadA5, and $\operatorname{arm} A$ for aminoglycoside resistance, $m p h(A)$ for macrolide resistance, cat $A 1$ for phenicol resistance, sull and sul2 for sulfonamide resistance, and $d f r A 17$ for trimethoprim resistance. The plasmid harbored class 1 integrons with a truncated $\operatorname{Tn} 3$ transposon with blaTEM-1C (Fig. 1; Table 4).

pECBHS_9_2 plasmid composition. The incompatible pECBHS_9_2 plasmid contained three origins of replication for IncFIA, IncFIB, and IncFII groups and conjugal transfer ( $t r a$ and $t r b$ ). It also contained $a a c$ (3)-IIa for aminoglycoside resistance, blaCTX-M-14 for $\beta$-lactam resistance, and tet (a) for tetracycline resistance. Along with drug resistance determinants, pECBHS_9_2 contained three toxin/antitoxin systems, including iucA, iucB, iucC_1, iucC_2, iucD, and iutA, associated with hydroxamate siderophore aerobactin synthesis.

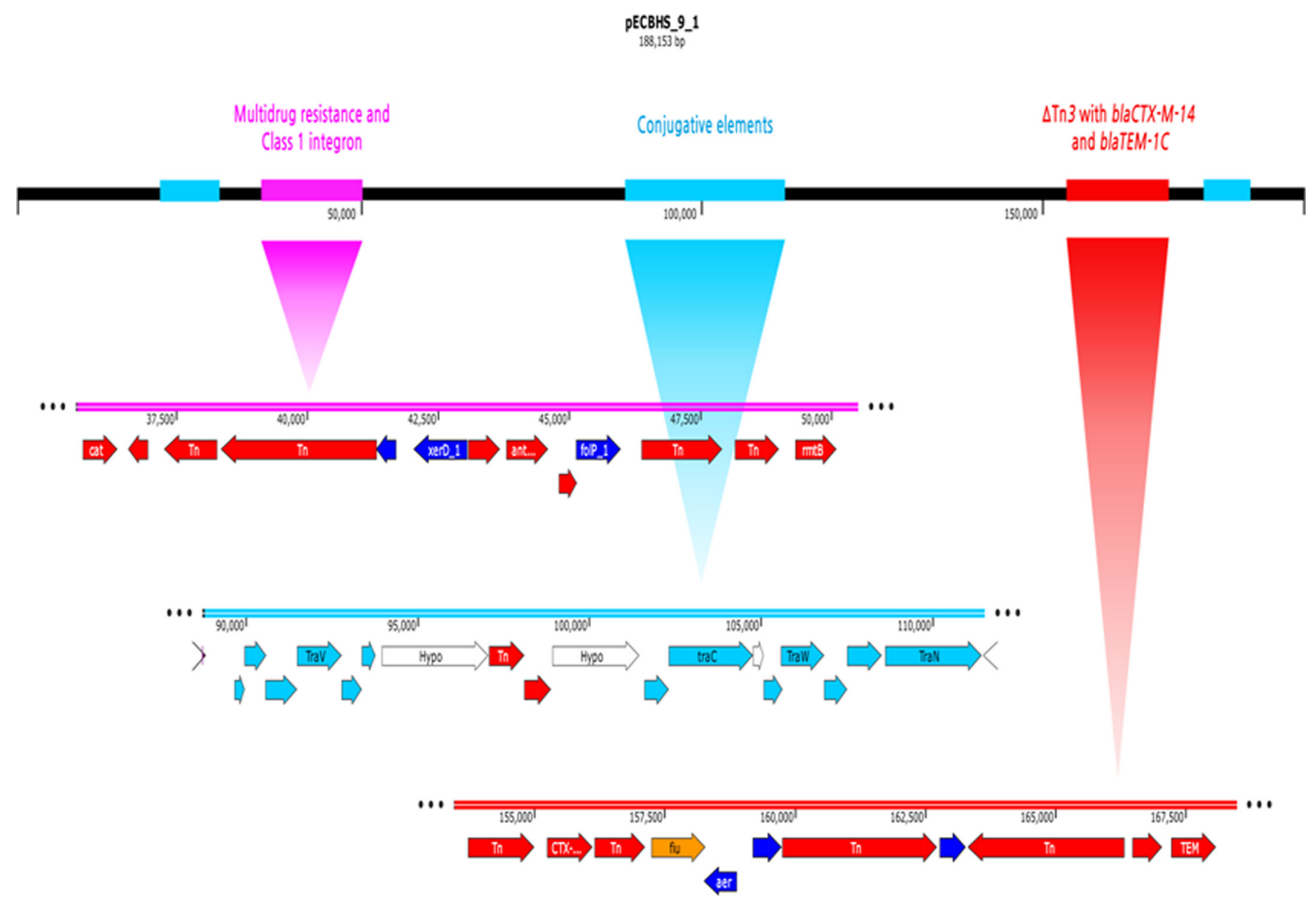

Fig. 1. Linear map of pECBHS_9_1. The IncA/C2 plasmid (pECBHS_9_1) harbored blaCTX-M-14 and blaTEM-1C for $\beta$-lactam resistance, aadA5, and armA for aminoglycoside resistance, $m p h(A)$ for macrolide resistance, cat 1 for phenicol resistance, sull, and sul2 for sulfonamide resistance, and $d f r A 17$ for trimethoprim resistance. The plasmid harbored Class 1 integrons containing a truncated Tn3 transposon with blaTEM-1C. Key: Pink line, Multidrug resistance and Class 1 integron; red line, $\mathrm{Tn} 3$ transposon; sky blue line, conjugative elements. 
Table 4. Antibiotic resistance genes and integrative conjugative elements in KPC-producing Enterobacteriaceae isolates.

\begin{tabular}{|c|c|c|c|c|c|c|c|c|c|c|c|}
\hline \multirow[b]{2}{*}{ Strain $^{\mathrm{a}}$} & \multicolumn{2}{|c|}{ Plasmid } & \multicolumn{8}{|c|}{ Antimicrobial resistance gene } & \multirow[b]{2}{*}{ Conjugants } \\
\hline & $\mathrm{pKPC}$ & Replicon & $\beta$-lactam & $\begin{array}{l}\text { Amino- } \\
\text { glycoside }\end{array}$ & $\begin{array}{l}\text { Tetra- } \\
\text { cycline }\end{array}$ & Macrolide & Phenicol & $\begin{array}{l}\text { Trime- } \\
\text { thoprim }\end{array}$ & $\begin{array}{l}\text { Sulpho- } \\
\text { namide }\end{array}$ & Quinolone & \\
\hline$\overline{\mathrm{CPEc} 171209}$ & blaKPC-2 & & $\begin{array}{l}\text { blaTEM-1, } \\
\text { blaCTX-M-1, } \\
\text { blaCTX-M-9, } \\
\text { blaSHV }\end{array}$ & $\operatorname{armA}$ & & & & & & & + \\
\hline${\underline{\mathrm{TCPEC}} 171209^{*}}^{*}$ & blaKPC-2 & & $\begin{array}{l}\text { blaTEM-1, } \\
\text { blaCTX-M-1, } \\
\text { blaCTX-M-9, } \\
\text { blaSHV }\end{array}$ & $\operatorname{armA}$ & & & & & & & \\
\hline pECBHS_9_1 & & IncA/C2 & $\begin{array}{l}\text { blaCTX-M-14, } \\
\text { blaTEM-1C }\end{array}$ & $\operatorname{aad} A 5, \operatorname{arm} A$ & & $m p h(A)$ & catA1 & $d f r A 17$ & sul1, sul2 & & \\
\hline pECBHS_9_2 & & $\begin{array}{l}\text { IncFIA, } \\
\text { IncFIB, } \\
\text { IncFII }\end{array}$ & blaCTX-M-14 & $\operatorname{aac}(3)-I I a$ & $\operatorname{tet}(A)$ & & & & & & \\
\hline pECBHS_9_5 & & IncX3 & $\begin{array}{l}\text { blaKPC-2, } \\
\text { blaSHV-182 }\end{array}$ & & & & & & & & \\
\hline СРКр171210 & blaKPC-2 & & $\begin{array}{l}\text { blaTEM-1, } \\
\text { blaCTX-M-1, } \\
\text { blaSHV }\end{array}$ & $r m t C$ & & & & & & $q n r B$ & + \\
\hline TCPKр171210 $^{*}$ & blaKPC-2 & & blaSHV & & & & & & & $q n r B$ & \\
\hline pKPBHS_10_1 & & $\operatorname{IncFIB}(\mathrm{K})$ & blaOXA-1 & $a a c\left(6^{\prime}\right)-I b-c r$ & $\operatorname{tet}(A)$ & & catB3 & dfrA14 & & $q n r B 1$ & \\
\hline pKPBHS_10_3 & & IncX3 & $\begin{array}{l}\text { blaKPC-2, } \\
\text { blaSHV-182 }\end{array}$ & & & & & & & & \\
\hline
\end{tabular}

${ }^{\mathrm{a}} \mathrm{CPEc} 171209$ and CPKp171210 are pre-conjugation strains, and TCPEc171209* and TCPKp171210* are the conjugated strains.

DNA sequencing was performed for strains that are underlined.

Whole-genome sequencing was performed for the indicated plasmids.
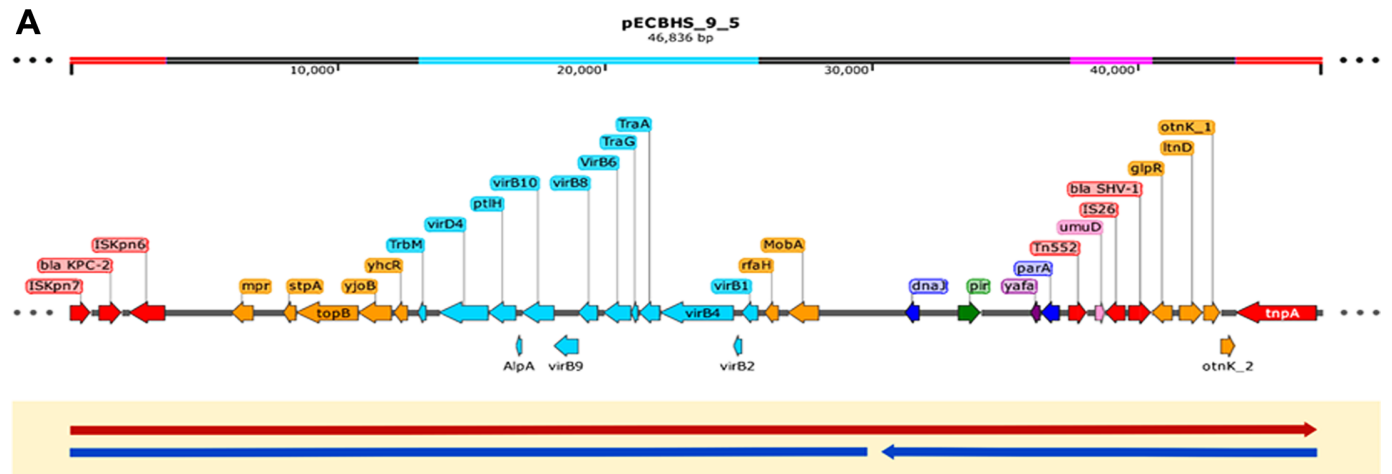

$29,145 . .29,593=449 \mathrm{bp}$

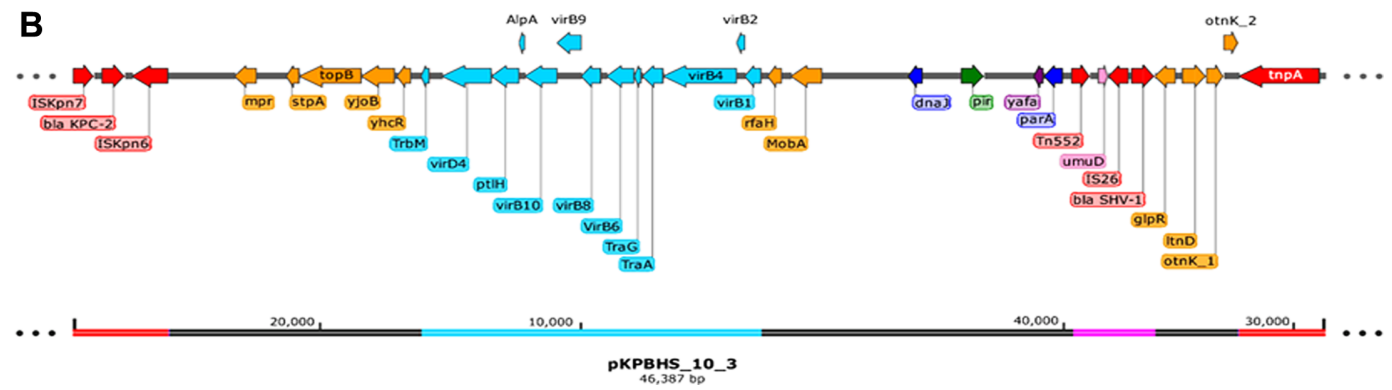

Fig. 2. Comparison of the blaKPC-2 regions of pECBHS 9 5 and pKPBHS_10_3. (A) Linear map of pECBHS_9_5 harboring $\triangle T$ Tn4401a with blaKPC-2, blaSHV-182, and conjugative elements. (B) Linear map of pKPBHS_10_3 harboring $\triangle \mathrm{Tn} 4401 a$ with blaKPC-2, blaSHV-182, and conjugative elements. The colored box indicates the gene. Most genes were well preserved but inverted. Genes are denoted by arrow colors based on the following gene function classification: green, plasmid replication; blue, plasmid stability; yellow, transcription and translation; sky blue, conjugative elements; pink, recombination and repair; red, antibiotic resistance; purple, other genes. Key: Pink line, blaSHV-182; red line, $\Delta \mathrm{Tn} 4401 a$ with blaKPC-2; sky blue line, conjugative elements. 


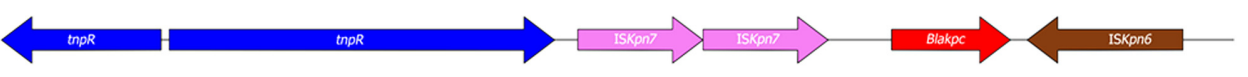

pECBHS_9_5

PKPBHS_10_3

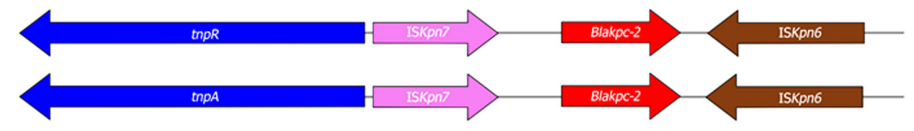

Fig. 3. Comparison of wild type Tn4401 and truncated forms in the plasmids pECBHS_9_5 and pKPBHS_10_3. Transposon Tn4401 is presented with direct repeats in each plasmid. Arrows in pink box, ISKpn7; arrows in brown box, ISKpn6; blue arrows, transposase; red arrows, blaKPC gene.

pECBHS_9_5 plasmid composition. The 46,836-bp IncX3 plasmid (pECBHS_9_5) belonged to an incompatibility group and harbored blaKPC-2 and blaSHV-182 for $\beta$-lactam resistance. The circular DNA had a $\mathrm{G}+\mathrm{C}$ content average of $48.0 \%$ and harbored 59 coding sequences and 25 annotated ORFs (Fig. 2). blaKPC-2 was located on a Tn 4401 variant designated as "isoform a," harboring a 99-bp deletion between blaKPC and istB [27]. DNA regions for plasmid replication $(r e p B)$ and stability ( $p a r A B$ and $s t p A)$, origin of transcription and translation $(r f a H$ and $y j o B)$, and conjugal transfer ( $t r a$ and $t r b$ ) were observed, and the plasmid harbored blaSHV-182 encoding a broad-spectrum $\beta$-lactamase (Fig. $2 \mathrm{~A}$ ).

\section{Sequencing and Annotation of CPKp171210}

The K. pneumoniae strain CPKp171210 genome comprised 5,760,457-bp, with a 5,478,640-bp chromosome and three plasmids. The G+C content average was $57.3 \%$. The 5,018 coding sequences comprised 86 tRNAs, 25 rRNAs, and 173 annotated ORFs. Unknown mutations in ompK37, ompK36, acrR, and $\operatorname{ramR}$ were observed. The chromosome encoded M.Kpn34618Dcm and type II restriction enzymes. Plasmid pKPBHS_10_1 M.EcoRII harbored a gene for Eco128I. The plasmid pKPBHS_10_2 contained restriction sites for type I restriction enzymes, S.Kpn1420II, and M.Kpn928I. CPKp171210 belonged to ST307, and the chromosome (CPKp171210) included fosA for fosfomycin resistance, blaSHV-28 for $\beta$-lactam resistance, and oqxA and $B$ for quinolone resistance.

pKPBHS_10_1 plasmid composition. The pKPBHS_10_1 plasmid comprised a 134,995-bp circular DNA with a $\mathrm{G}+\mathrm{C}$ content of $52.0 \%$ on an average and 56 annotated ORFs. Plasmid pKPBHS_10_1 contained M.EcoRII and Eco128I type II restriction enzymes. pKPBHS_10_1 is an IncFIB(K) plasmid which harbored blaOXA-1 for $\beta$-lactam resistance, $a a c\left(6^{\prime}\right)$-Ib-cr for aminoglycoside resistance, $q n r B 1$ for fluoroquinolone resistance, catB3 for phenicol resistance, tet $(A)$ for tetracycline resistance, and $d f r A 14$ for trimethoprim resistance. Plasmid pKPBHS_10_1 harbored CusCFBA genes for mediating resistance to copper and silver.

pKPBHS_10_3 plasmid composition. The 46,387-bp incompatible IncX3 plasmid (pKPBHS_10_3) harbored blaKPC-2 and blaSHV-182 for $\beta$-lactam resistance. This plasmid had a $\mathrm{G}+\mathrm{C}$ content average of $48.0 \%$ and harbored 56 coding sequences and 38 annotated ORFs (Fig. 2B). blaKPC-2 was located on a variant of Tn4401, called "isoform a," that contained a 99-bp deletion between blaKPC and istB. DNA regions for plasmid replication $(r e p B)$ and stability ( $p a r A B$ and $s t p A)$, origin of transcription and translation $(r f a H$ and $y j o B)$, and conjugal transfer ( $t r a$ and $t r b$ ) were detected, along with blaSHV-182 encoding a broad-spectrum $\beta$-lactamase (Fig. 2B).

Comparison of the Composition of pECBHS_9_5 and pKPBHS_10_3

Plasmid pECBHS_9_5 was 449 bp longer than plasmid pKPBHS_10_3. These two plasmids had 99\% sequence similarity, except for the extra $449 \mathrm{bp}$ in pECBHS_9_5. Alignment analyses between plasmid pKPBHS_10_3 and pECBHS_9_5 revealed $100 \%$ identity to the blaKPC-2 allele (Fig. 2). Both plasmids were IncX3 incompatible plasmids harboring blaKPC-2 and blaSHV-182 $\beta$-lactam resistance genes. These plasmids had an average $\mathrm{G}+\mathrm{C}$ content of $48.0 \%$ and could be transferred to the recipient $E$. coli J53 strain through surface conjugation (Table 3 ). blaKPC-2 was located on the Tn 4401 "isoform a" variant (Fig. 3).

\section{Discussion}

In this study, two clinical isolates, KPC-2 E. coli and KPC-2 K. pneumoniae, were obtained from rectal swabs. Recent studies on E. coli ST410 have suggested this stain as another successful pandemic extraintestinal pathogenic E. coli strain, with a lineage similar to that of ST131 [8]. Our results are largely concurrent with those of Roer et al. [10], with the E. coli (CPEc171209) ST410 strain belonging to the serotype O8:H21 containing antibacterial clade $\mathrm{C} 4 / \mathrm{H} 24$ [10]. Roer et al. [10] reported that the E. coli ST410 lineage persists and causes recrudescent infections, such as hematological infections, in humans. ST410 has high worldwide prevalence and is widely distributed in the bloodstream, indicating that it can be transmitted between patients and cause hospital outbreaks [10]. K. pneumoniae (CPKp171210) ST307 harbors unknown mutations in ompK36 and ompK37. In whole-genome sequencing analysis, these mutations were considered deletion mutations in DNA sequences. Isolates harboring blaKPC and expressing ompK36 reportedly have lower carbapenem MICs. Furthermore, alterations in outer membrane proteins are reportedly associated with increased MICs of carbapenems [23]. Herein, both strains exhibited high or intermediate resistance to all tested antimicrobial agents, except for amikacin, tigecycline, and colistin. Resistance to a particular antibiotic can affect resistance to another antibiotic. 
For example, cross-resistance, such as those between cephalosporin and $\beta$-lactam, quinolone and methicillinresistant Staphylococcus aureus, and macrolide and pneumococcal resistance, have been reported. MDR E. coli CPEc171209 and K. pneumoniae CPKp171210 also cause cross-resistance to various antibiotics (Table 1).

The genes responsible for conjugal transfer of the IncX3 plasmid include tra/trb clusters and pil genes coding the type IV pilus. The IncX3 type is a predominant plasmid associated with blaNDM-1 [28] and occasionally harbors blaKPC [29], often accompanying a second plasmid including IncFII and ColE types [30].

Plasmid pECBHS_9_5 of CPEc171209 harbored blaKPC-2 bracketed by the Tn3-type transposon Tn4401 to pKPBHS_10_3 of CPKp171210 (Figs. 2A and 2B). The blaKPC-2 gene was located within a truncated Tn4401 transposon; Tn 4401 harbors the $\operatorname{tnp} A$ gene encoding a transposase, the $\operatorname{tn} p R$ gene encoding a resolvase, and two insertion sequence elements, ISKpn7 and ISKpn6, located at each end of the blaKPC gene [31]. Furthermore, both CPEc171209 and CPKp171210 harbored transconjugation factors and the Tn3-type transposon, potentially resulting in cross-infection and horizontal migration. Therefore, the risk of interspecies mobility and infection is high. Plasmid pECBHS_9_2 harbors genes encoding virulence factors and toxin/antitoxin systems, which results in the enhancement of bacterial fitness in human hosts and prolonged persistence [32]. KPC-2 E.coli and KPC-2 K. pneumoniae were continuously monitored by rectal swab detection over the course of 3 months until the patient died. Plasmids pKPBHS_10_1 and pKPBHS_10_2 harbor various genes related to the resistance against diverse kinds of antimicrobial agents and defend themselves through type I and II restriction enzymes. The pKPBHS_10_1 plasmid also harbors CusCFBA proteins mediating resistance to copper and silver through cation efflux. Gram-negative bacteria, including E. coli frequently use tripartite efflux complexes of the resistancenodulation cell division superfamily transporters to pump out diverse toxic compounds [33, 34]. The efflux system CusCFBA is liable for removing biocidal $\mathrm{Cu}(\mathrm{I})$ and $\mathrm{Ag}(\mathrm{I})$ ions $[35,36]$, and the Cus determinant of gramnegative bacteria encodes CusCFBA proteins. CusA and CusB are essential for copper resistance, while CusC and CusF are required for overall resistance.

This study investigated a case of persistent infection in a patient because of interspecies migration of carbapenem-resistant Enterobacteriaceae caused by an E. coli ST410 strain. Molecular genetic evidence obtained herein reveals the nature of this occurrence and indicates the risk of ST410 E. coli infections. The emergence of KPC-producing E. coli is concerning because the blaKPC-2-bearing plasmid may result in interspecies propagation of resistance. E. coli ST410 represents a globally distributed lineage and is responsible for diverse antimicrobial resistance determinants, including extended-spectrum $\beta$-lactamases, pAmpCs, carbapenemases, and colistin resistance genes. Furthermore, the present results suggest that ST410 is a high-risk bacterium when it infects the host. Common sequences of virulence factors were observed in plasmids in ST410 CPEc171209 and in another high-risk group, ST307 CPKp1210, indicating the potential for cross-transmission, thus limiting treatment alternatives and maintaining long-term pathogenicity.

Carbapenem-resistant bacteria can undergo transgenic translocation and may spread rapidly via horizontal migration in patients. Together with its demonstrated high trainability among patients, E. coli ST410 poses an elevated risk in clinical settings; therefore, it should be considered as a lineage with emerging "high-risk" clones and be closely monitored. Although the number of patients analyzed in this study was limited (1 patient), this study clearly elucidates the propagation pattern of KPC-producing E. coli (ST410) strains, which frequently occur in South Korea. Further investigation of the molecular genetics of KPC-producing E. coli (ST410) are needed.

\section{Acknowledgment}

We would like to thank the BHS Hanseo Hospital for the kind gesture of accommodating us and for allowing us to continue our study.

\section{Conflict of Interest}

The authors have no financial conflicts of interest to declare.

\section{References}

1. Rhomberg PR, Deshpande LM, Kirby JT, Jones RN. 2007. Activity of meropenem as serine carbapenemases evolve in US Medical Centers: monitoring report from the MYSTIC Program (2006). Diagn. Microbiol. Infect. Dis. 59: 425-432.

2. Munoz-Price LS, Poirel L, Bonomo RA, Schwaber MJ, Daikos GL, Cormican M, et al. 2013. Clinical epidemiology of the global expansion of Klebsiella pneumoniae carbapenemases. Lancet Infect. Dis. 13: 785-796.

3. Pitout JD, Nordmann P, Poirel L. 2015. Carbapenemase-producing Klebsiella pneumoniae, a key pathogen set for global nosocomial dominance. Antimicrob. Agents Chemother. 59: 5873-5884.

4. Adler A, Miller-Roll T, Assous MV, Geffen Y, Paikin S, Schwartz D, et al. 2015. A multicenter study of the clonal structure and resistance mechanism of KPC-producing Escherichia coli isolates in Israel. Clin. Microbiol. Infect. 21: 230-235.

5. Piazza A, Caltagirone M, Bitar I, Nucleo E, Spalla M, Fogato E, et al. 2016. Emergence of Escherichia coli sequence type 131 (ST131) and ST3948 with KPC-2, KPC-3 and KPC-8 carbapenemases from a long-term care and rehabilitation facility (LTCRF) in northern Italy. Adv. Exp. Med. Biol. 901: 77-89.

6. Chavda KD, Chen L, Jacobs MR, Bonomo RA, Kreiswirth BN. 2016. Molecular diversity and plasmid analysis of KPC-producing Escherichia coli. Antimicrob. Agents Chemother. 60: 4073-4081.

7. Xu G, Jiang Y, An W, Wang H, Zhang X. 2015. Emergence of KPC-2-producing Escherichia coli isolates in an urban river in Harbin, China. World J. Microbiol. Biotechnol. 31: 1443-1450.

8. Schaufler K, Semmler T, Wieler LH, Wöhrmann M, Baddam R, Ahmed N, et al. 2016. Clonal spread and interspecies transmission of clinically relevant ESBL-producing Escherichia coli of ST410_-another successful pandemic clone? FEMS Microbiol. Ecol. 92 : fiv155.

9. Falgenhauer L, Imirzalioglu C, Ghosh H, Gwozdzinski K, Schmiedel J, Gentil K, et al. 2016. Circulation of clonal populations of fluoroquinolone-resistant CTX-M-15-producing Escherichia coli ST410 in humans and animals in Germany. Int. J. Antimicrob. Agents 47: 457-465. 
10. Roer L, Overballe-Petersen S, Hansen F, Schønning K, Wang M, Røder BL, et al. 2018. Escherichia coli sequence type 410 is causing new international high-risk clones. mSphere 3: e00337-18.

11. Solgi H, Badmasti F, Aminzadeh Z, Giske CG, Pourahmad M, Vaziri F, et al. 2017. Molecular characterization of intestinal carriage of carbapenem-resistant Enterobacteriaceae among inpatients at two Iranian university hospitals: first report of co-production of blaNDM-7 and blaOXA-48. Eur. J. Clin. Microbiol. Infect. Dis. 36: 2127-2135.

12. Ohno Y, Nakamura A, Hashimoto E, Matsutani H, Abe N, Fukuda et al. 2017. Molecular epidemiology of carbapenemase-producing Enterobacteriaceae in a primary care hospital in Japan, 2010-2013. J. Infect. Chemother. 23: 224-229.

13. He S, Chandler M, Varani AM, Hickman AB, Dekker JP, Dyda F. 2016. Mechanisms of evolution in high-consequence drug resistance plasmids. MBio 7: e01987-16.

14. Mouloudi E, Protonotariou E, Zagorianou A, Iosifidis E, Karapanagiotou A, Giasnetsova T, et al. 2010. Bloodstream infections caused by metallo-beta-lactamase/Klebsiella pneumoniae carbapenemase-producing K. pneumoniae among intensive care unit patients in Greece: risk factors for infection and impact of type of resistance on outcomes. Infect. Control Hosp. Epidemiol. 31: 12501256.

15. Walsh TR, Weeks J, Livermore DM, Toleman MA. 2011. Dissemination of NDM-1 positive bacteria in the New Delhi environment and its implications for human health: an environmental point prevalence study. Lancet Infect. Dis. 11: 355-362.

16. Copur Cicek A, Ozad Duzgun A, Saral A, Sandalli C. 2014. Determination of a novel integron-located variant (blaOXA-320) of Class D $\beta$-lactamase in Proteus mirabilis. J. Basic Microbiol. 54:1030-1035.

17. Clinical and Laboratory Standards Institute. 2018. Methods for dilution antimicrobial susceptibility tests for bacteria that grow aerobically. Clinical and Laboratory Standards Institute, Wayne, P. A.

18. EUCAST. Antimicrobial susceptibility testing of bacteria. Available from http://www.eucast.org/ast_of_bacteria/ (Updated on Jan 2017). Accessed April 30, 2019.

19. Jeong S, Kim JO, Jeong SH, Bae IK, Song W. 2015. Evaluation of peptide nucleic acid-mediated multiplex real-time PCR kits for rapid detection of carbapenemase genes in gram-negative clinical isolates. J. Microbiol. Methods 113: 4-9.

20. Pérez-Pérez FJ, Hanson ND. 2002. Detection of plasmid-mediated AmpC b-lactamase genes in clinical isolates by using multiplex PCR. J. Clin. Microbiol. 40: 2153-2162.

21. Ryoo NH, Kim EC, Hong SG, Park YJ, Lee K, Bae IK, et al. 2005. Dissemination of SHV-12 and CTX-M-type extended-spectrum beta-lactamases among clinical isolates of Escherichia coli and Klebsiella pneumoniae and emergence of GES-3 in Korea. J. Antimicrob. Chemother. 56: 698-702.

22. Yamane K, Wachino J, Suzuki S, Arakawa Y. 2008. Plasmid-mediated qepA gene among Escherichia coli clinical isolates from Japan. Antimicrob. Agents Chemother. 52: 1564-1566.

23. Landman D, Bratu S, Quale J. 2009. Contribution of ompK36 to carbapenem susceptibility in KPC-producing Klebsiella pneumoniae. J. Med. Microbial. 58: 1303-1308.

24. Wirth T, Falush D, Lan R, Colles F, Mensa P, Wieler LH, et al. 2006. Sex and virulence in Escherichia coli: an evolutionary perspective. Mol. Microbiol. 60: 1136-1151.

25. Diancourt L, Passet V, Verhoef J, Grimont PA, Brisse S. 2005. Multilocus sequence typing of Klebsiella pneumoniae nosocomial isolates. J. Clin. Microbiol. 43: 4178-182.

26. Jeong SH, Lee KM, Lee J, Bae IK, Kim JS, Kim HS, et al. 2015. Clonal and horizontal spread of the blaOXA-232 gene among Enterobacteriaceae in a Korean hospital. Diagn. Microbiol. Infect. Dis. 82: 70-72.

27. Naas T, Cuzon G, Truong HV, Nordmann P. 2012. Role of ISKpn7 and deletions in blaKPC gene expression. Antimicrob. Agents Chemother. 56: 4753-4759.

28. Yang Q, Fang L, Fu Y, Du X, Shen Y, Yu Y. 2015. Dissemination of NDM-1-producing Enterobacteriaceae mediated by the IncX3-type plasmid. PLoS One 10: e0129454.

29. Chen L, Chavda KD, Melano RG, Jacobs MR, Levi MH, Bonomo RA, et al. 2013. Complete sequence of a bla (KPC-2)-harboring IncFII (K1) plasmid from a Klebsiella pneumoniae sequence type 258 strain. Antimicrob. Agents. Chemother. 57: 1542-1545.

30. Kassis-Chikhani N, Frangeul L, Drieux L, Sengelin C, Jarlier V, Brisse S, et al. 2013. Complete nucleotide sequence of the first KPC-2and SHV-12-encoding IncX plasmid, pKpS90, from Klebsiella pneumoniae. Antimicrob. Agents Chemother. 57: 618-620.

31. Naas T, Cuzon G, Villegas MV, Lartigue MF, Quinn JP, Nordmann P. 2008. Genetic structures at the origin of acquisition of the betalactamase blaKPC gene. Antimicrob. Agents Chemother. 52: 1257-1263.

32. Jeong S, Kim JO, Yoon EJ, Bae IK, Lee W, Lee H, et al. 2018. Extensively drug-resistant Escherichia coli sequence type 1642 carrying an IncX3 plasmid containing the blaKPC-2 gene associated with transposon Tn4401a. Ann. Lab. Med. 38: 17-22.

33. Tseng TT, Gratwick KS, Kollman J, Park D, Nies DH, Goffeau A, et al. 1999. The RND permease superfamily: an ancient, ubiquitous, and diverse family that includes human disease and development protein. J. Mol. Microbiol. Biotechnol. 1: 107-125.

34. Nies DH. 2003. Efflux-mediated heavy metal resistance in prokaryotes. FEMS Microbiol. Rev. 27: 313-339.

35. Franke S, Grass G, Nies DH. 2001. The product of the $y b d E$ gene of the Escherichia coli chromosome is involved in detoxification of silver ions. Microbiology 147: 965-972.

36. Franke S, Grass G, Rensing C, Nies DH. 2003. Molecular analysis of the copper-transporting efflux system CusCFBA of Escherichia coli. J. Bacteriol. 185: 3804-3812. 